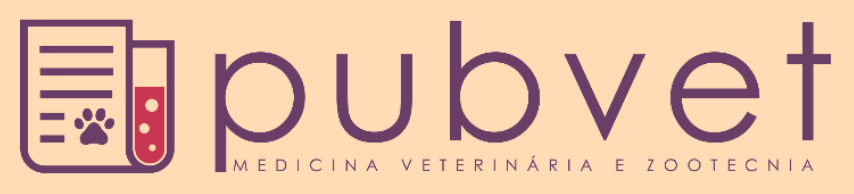

https://doi.org/10.31533/pubvet.v13n01a265.1-6

\title{
Sistemas agroflorestais como alternativa agroecológica: Revisão
}

\author{
Éder Bruno Rebelo da Silva ${ }^{1 * \bullet}$, Welligton Conceição da Silva ${ }^{2} \bullet$, Eudilene Dalet Vitor de Sousa ${ }^{1}$ \\ $\bullet$, Ana Paula da Cruz Gato ${ }^{\circ}{ }^{\circ}$, Lennon Júnior Silva Araújo ${ }^{3 \odot}$ \\ ${ }^{I}$ Graduando em Agronomia pelo Centro Universitário Luterano de Santarém (CEULS/ULBRA), Av. Sergio Heinz, n.1787, CEP. 6805-000, Santarém, Pará, Brasil. \\ ${ }^{2}$ Médico Veterinário, Mestrando pelo programa de Pós-Graduação em Produção e Saúde Animal pela Universidade Federal Rural da \\ Amazônia (UFRA), Estrada principal da UFRA, curió Utinga, CEP. 2150-2476, Belém, Pará, Brasil. \\ ${ }^{3}$ MédicoVeterinário, Autônomo, Avenida São Sebastião 1126, CEP. 68005-090, Santarém, Pará, Brasil. \\ *Autor para correspondência, E-mail: eder.b.rebelo@gmail.com
}

Resumo. O objetivo nesse trabalho é explorar o potencial de implantação e enriquecimento de um sistema agroflorestal (SAF) em uma propriedade de base agroecológica, no munícipio de Santarém, implementado com técnicas e arranjos especiais para se obter aumento da produtividade e biodiversidade, consequentemente produzindo de maneira consciente e agredido menos o ecossistema local. As florestas que recobrem o planeta apresentam grande riqueza vegetal, com diversos efeitos benéficos sobre o clima e recursos hídricos, resultando da grande biomassa e da fixação de carbono. Através dos SAF's criamse diferentes estratos ou andares vegetais, procurando imitar uma floresta natural, onde as culturas agrícolas e vegetação influenciam no processo de ciclagem de nutrientes e no aproveitamento da energia solar são considerados os elementos estruturais básicos e principais para a estabilidade do sistema. Dessa forma, a implantação de sistemas agroflorestais em propriedades rurais pode maximizar a escala produtiva em pequenas áreas, ampliando os processos físicos, químicos e biológicos nos agroecossistemas, onde o sistema agroflorestal foi implantado.

Palavras-chave: agroecologia, culturas agrícolas, propriedade rural, sustentabilidade

\section{Agroforestry systems as an agroecological alternative: Review}

Abstract. The objective of this work is to explore the potential of implantation and enrichment of a Saf in an agroecological-based property in the municipality of Santarém, implementing with special techniques and arrangements to increase productivity and biodiversity, consequently producing less aggressively and less aggressively the local ecosystem. The forests that cover the planet present great vegetal richness, with diverse beneficial effects on the climate and water resources, resulting from the great biomass and the fixation of carbon. Through SAF's different strata or plant stages are created, trying to imitate a natural forest, where the agricultural crops and vegetation influence the process of nutrient cycling and the use of solar energy are considered the basic and main structural elements for stability of the system. In this way, the implantation of Agroforestry Systems in rural properties can maximize the productive scale in small areas, expanding the physical, chemical and biological processes in the agroecosystems, where the Agroforestry System was implanted.

Keywords: agroecology, agricultural crops, rural property, sustainability 


\title{
Sistemas agroforestales como alternativa agroecológica: Revisión
}

\begin{abstract}
Resumen. El objetivo en este trabajo es explorar el potencial de implantación y enriquecimiento de un sistema agroforestal (SAF) en una propiedad de base agroecológica, en el municipio de Santarém, implementado con técnicas y arreglos especiales para obtener aumento de la productividad y biodiversidad, consecuentemente produciendo de manera consciente y agrediendo menos el ecosistema local. Los bosques que cubren el planeta presentan gran riqueza vegetal, con diversos efectos beneficiosos sobre el clima y recursos hídricos, resultando de la gran biomasa y de la fijación de carbono. A través de los SAF se crean diferentes estratos o pisos vegetales, buscando imitar un bosque natural, donde los cultivos agrícolas y vegetación influencian en el proceso de ciclo de nutrientes y en el aprovechamiento de la energía solar se consideran los elementos estructurales básicos y principales para la estabilidad del sistema. De esta forma, la implantación de sistemas agroforestales en propiedades rurales puede maximizar la escala productiva en pequeñas áreas, ampliando los procesos físicos, químicos y biológicos en los agroecosistemas, donde el sistema agroforestal fue implantado.
\end{abstract}

Palabras clave: agroecología, cultivos agrícolas, propiedad rural, sostenibilidad

\section{Introdução}

As florestas que recobrem o planeta apresentam grande riqueza vegetal, com diversos efeitos benéficos sobre o clima e recursos hídricos, resultando da grande biomassa e da fixação de carbono. Dados apresentados pela EMBRAPA (2013) apontam que mais de 15\% dos solos do mundo encontramse degradados, sendo que $98 \%$ dessa área está relacionada a produção primária.

Os sistemas agroflorestais (SAF's) apresentam-se como possibilidade sustentável de uso da terra, na Amazônia surge com as culturas indígenas, caboclas e ribeirinhas se desde então atendem as necessidades de evolução no uso da terra, especialmente em regiões tropicais, aumentando a biodiversidade com o intuito de atender as necessidades das comunidades (Daniel et al., 1999). Mesquita Filho et al. (2006) afirmaram que um sistema agroflorestal leva 40 anos para cultivar uma floresta de alimentos, o que a natureza levaria em torno de 100 anos.

Em oposição aos sistemas agroflorestais, os cultivos convencionais apresentam vários impactos ambientais e sociais, gerando problemas como o aumento da emissão de gases do efeito estufa, aumento do desmatamento e da poluição de solos e das águas (Canuto, 2017; Pereira, 2004). Uma alternativa que se mostra desafiadora, inovadora e eficaz é a implantação de SAF's, que visam aumentar a sustentabilidade dos agroecossistemas (Canuto, 2017; Ribaski et al., 2001). Os cultivos em SAF's estão relacionados ao melhor aproveitamento da área e a maior produção total por módulo de trabalho. Esses sistemas partem da premissa de otimizar as áreas produtivas por meio do uso de diferentes extratos vegetais com base no ciclo natural das plantas, obedecendo às características de cada espécie no modelo agroflorestal (Rippstein et al., 2001). Nesse contexto, a agroecologia se mostra enquanto ciência destinada a apoiar a transição de modelos de agriculturas convencionais para modelos sustentáveis como é o caso dos Saf's.

O objetivo deste trabalho foi apresentar a importância da implantação dos sistemas agroflorestais em propriedades rurais.

\section{Agricultura alternativa}

É o conjunto de práticas e técnicas de cultivo de terra que diferente da agricultura tradicional, tem como objetivos o desenvolvimento sustentável e a produção de gêneros agrícolas sem qualquer contaminação por agrotóxicos ou pesticidas (Penteado, 2007; Penteado, 2016), é também conhecida como agricultura ecológica ou agricultura verde, visando sempre o equilíbrio ecológico na lavoura, buscando conservar as características naturais do solo e manter em equilíbrio os ciclos biológicos e as interações biológicas, ademais prioriza a relação entre o homem e o meio natural. A agricultura alternativa tem como objetivo produzir alimentos em maior escala e de melhor qualidade respeitando o 
meio ambiente e a saúde humana, criando soluções adequadas para atingir as causas e não os sintomas, valorizando o homem e seu trabalho, garantido a fertilidade do solo com a integração da lavoura, criação de animais e floresta. Segundo Stoate et al. (2009) a agricultura alternativa surgiu na segunda década do século XIX pela agricultura biodinâmica. A agricultura alternativa não utiliza métodos que possam contaminar ou degradar o solo, visando sempre o equilíbrio ecológico, práticas como queimadas não são agregadas aos processos agrícolas, não realiza desmatamentos para geração ou ampliação de áreas agricultáveis emprega fertilizante se métodos naturais de controle de pragas, dessa forma, preservando o solo, ecossistemas, recursos naturais e produzindo alimentos sem contaminação química (Penteado, 2016).

A eficiência de qualquer sistema alternativo de produção deve ser baseada em determinados princípios. De acordo com Oliveira et al. (2012) esses princípios são:

- Ecologicamente sustentável: deve-se aumenta a utilização de recursos naturais, através de técnicas adequadas para que os sistemas de produção se tornem ecologicamente sustentáveis preservando os recursos naturais e produzindo alimentos saudáveis;

- Socialmente justa: a tecnologia deve ser benéfica e apropriada a qualquer um, alcançando e suprindo as necessidades dos produtores e entregando produtos de qualidade aos consumidores;

- Tecnologicamente adequada: solo e clima são diferentes em várias regiões e para cada situação, produção e manejo e necessário se criar uma técnica apropriada para a produção;

- Economicamente viável: é preciso utilizar técnicas que otimizem a produção e consequentemente acarrete um retorno financeiro e uma economia produtiva para compensar os investimentos;

- Culturalmente aceito: cada região tem suas peculiaridades que precisam ser respeitadas quando se pensa em técnicas de desenvolvimento sustentável.

\section{Aplicação prática de técnicas alternativas}

Em vários setores agrícolas estão sendo executadas diversas tecnologias alternativas de produção, após a revolução verde o homem passou a melhorar as espécies vegetais, segundo Oliveira \& Oswaldo (2012) nossos solos precisam de manejo adequado que mantenha e melhore as características físicas, biológicas e químicas que evite problemas com compactação, erosão e degradação da matéria orgânica, com esse manejo adequado é possível reduzir a incidência de pragas, doenças e plantas daninhas e consequentemente melhorar a produção. Utiliza-se, portanto:

- Cobertura morta: promove estabilidade térmica, controlam plantas daninhas e evita a erosão por impacto de gotas de chuva, favorece a biologia do solo e mantém a umidade;

- Adubação verde: além dos citados acima, fixa o nitrogênio (leguminosas), controla pragas (nematóides), mobilizam nutrientes, interrompe o ciclo de pragas, além da ação alelopáticas sobre plantas daninhas. As mais usadas são: crotalária, feijão de porco, guandu, mucuna preta e soja perene;

- Consorciação: otimiza o aproveitamento de água, luz solar e nutrientes. Possibilita o trabalho com plantas atrativas, que atrai para perto de si as pragas e repelentes que repelem as pragas;

- Rotação de culturas: reduz o aparecimento de doenças, a infestação de ervas daninhas e pragas, aumenta a disponibilidade de nutrientes no solo, diminuindo a necessidade de compra de fertilizantes.

\section{Sistemas agroecológicos}

A agroecologia é o termo principal do que hoje é chamada de agricultura sustentável. Segundo Altieri (1998) trata-se de uma nova abordagem que integra os princípios agronômicos, ecológicos, 
socioeconômicos sobre a compreensão e avaliação do efeito das tecnologias sobre os sistemas agrícolas e a sociedade como um todo incluindo dimensões ecológicas, sociais e culturais.

Para o MAPA é uma abordagem que integra os conhecimentos científicos aos conhecimentos populares, para compreensão, avaliação e implantação de sistemas agrícolas com vistas à sustentabilidade. Surgiu em meados da década de 70 tem como princípio básico elaborar uma relação racional com os recursos naturais.

Os princípios ecológicos são observados em diversas ramificações, que juntas formam a árvore agroecológica, são elas: Agricultura Orgânica e Biológica, Agricultura Biodinâmica, Agricultura Natural e Permacultura. Santana et al. (2003) justificam que sistemas agroecológicos são ferramentas que garantem a sustentabilidade e produtividade do agroecossistema oriundos dos estilos de produção vigente no decorrer da história. Para Leff (2002) a agroecologia possibilita mudanças que podem converter os recursos agrícolas e florestais em bases de desenvolvimento e bem-estar das comunidades rurais parece, também, como um meio para a proteção efetiva do meio ambiente, da biodiversidade e do equilíbrio ecológico do planeta.

A política nacional de agroecologia foi instituída em 2012, no decreto $\mathrm{n}^{\circ} 7.794$ com objetivo de articular e adequar às várias políticas, programas e ações de desenvolvimento no âmbito do governo federal, que visam introduzir a transição agroecológica e fomentar a produção em sistemas agroecológicos, contribuindo para a produção sustentável e de alimentos saudáveis, dessa forma, aliando o desenvolvimento rural com a conservação dos recursos naturais.

\section{Agroecossistemas}

Segundo Gliessman (2005), o agroecossistema é o conjunto de todos os organismos, sejam eles de interesse agropecuário ou não, considerando as interações nos níveis de população, comunidade e ecossistema, tendo como prioridade a sustentabilidade. Para (Conway, 1987), os agroecossistema são sistemas ecológicos modificados pelo ser humano para produzir comida, fibra ou outro produto agrícola. A modificação dos sistemas naturais pelo homem, para manejo agrícola e produção de bens necessários à sua sobrevivência, formam o agroecossistema, com essa intervenção humana, o controle natural e os próprios mecanismo são substituídos pelos artificiais, a qual o produtor se encarrega de manejar. Para Altieri et al. (2015) em um agroecossistema a produção agroecológica sustentável, deriva principalmente do equilíbrio entre as espécies vegetais, solos, nutrientes, luz solar e organismos que compõe o agrossistema de produção, dessa forma, colocam-se em evidência um pilar essencial de estratégia agroecológica, produzir sem agredir e aumentando a biodiversidade. A interação entre elementos formadores do ecossistema apresenta efeitos benéficos, são eles:

1- cria uma cobertura vegetal continua para a proteção do solo;

2- assegura constate produção de alimentos;

3- fecha os ciclos de nutrientes e garante o uso eficaz dos recursos locais;

4- contribui para a conservação do solo e dos recursos hídricos;

5- intensifica o controle biológico de pragas fornecendo habitat para os inimigos naturais (Altieri, 1998).

\section{SAF's - Sistemas agroflorestais}

Os SAF's são reconhecidamente modelos de exploração de solos que mais se aproximam ecologicamente da floresta natural e que, por isso, são considerados como importante alternativa de uso sustentável do ecossistema tropical úmido (Bandy et al., 1994), ou seja, são sistemas de produção agrícola que consorciam espécies florestais (frutíferas e/ou madeireiras) com cultivos agrícolas e em alguns casos também animais, na mesma área e numa sequência temporal.

Atualmente na literatura diversos conceitos sobre SAF's são empregados, o que pode causar confusão no entendimento do sistema, entretanto agroflorestal é um termo novo, para uma prática muito antiga utilizada pelos indígenas. Segundo King et al. (1978), SAF’s são sistemas sustentáveis de uso de terra combinam de maneira simultânea ou em sequência, a produção de cultivos agrícolas com 
plantações de árvores frutíferas nativas e exóticas ou espécies florestais de valor econômico ou ecológico presente na região, utilizando a mesma unidade de terra e aplicando técnicas de manejo que são compatíveis com as práticas culturais da população local. Os Saf's têm grande potencial para desenvolvimento sustentável, através da conservação das águas e solos, possibilitando uma adequação a pequena produção, recuperação de fragmentos e matas ciliares, além da conservação da biodiversidade (Valeri et al., 2003).

Pelo SAF's criam-se diferentes estratos ou andares vegetais, procurando imitar uma floresta natural, onde as culturas agrícolas e vegetação influenciam no processo de ciclagem de nutrientes e no aproveitamento da energia solar são considerados os elementos estruturais básicos e principais para a estabilidade do sistema. Os SAFs seguem os princípios agroecológicos e têm por objetivo harmonizar os agroecossistema com os processos dinâmicos dos ecossistemas naturais, buscando-se assim, o oposto da agricultura moderna, onde o homem buscar adaptar as plantas e ecossistemas as necessidades contemporâneas. Assim, pelo SAFs obtém-se uma importante ferramenta para a agricultura familiar que em consorcio com a agroecologia e suas técnicas ajudam na agricultura familiar e na conservação dos recursos naturais.

Os sistemas agroflorestais são considerados alternativos de uso de recursos naturais que causam pouca e/ou nenhuma degradação ao meio ambiente, principalmente por respeitarem os princípios básicos de manejo sustentável agro-ecossistema (Canuto, 2017; Macedo, 2010; Ribaski et al., 2001). Assim, a principal vantagem dos SAF's em comparação ao sistema convencional relacionado ao uso do solo e restauração ambiental torna-se uma potência dentro dos recursos naturais por promover melhorias na utilização de energia solar por promover a ciclagem de nutrientes e proteção ao solo contra lixiviação e erosão. Os Saf's têm grande potencial para desenvolvimento sustentável, através da conservação das águas e solos, possibilitando uma adequação a pequena produção, recuperação de fragmentos e matas ciliares, além da conservação da biodiversidade (Valeri et al., 2003).

\section{Conclusão}

Diante do exposto, pode se concluir que os sistemas agroflorestais apresentam características favoráveis para aumentar a produção em pequenas áreas. Os SAF's proporcionam a recuperação de áreas saturadas pela monocultura, simular uma floresta natural que buscar melhorar fatores abióticos e bióticos auxiliando na ciclagem de nutrientes e consequentemente melhorar os processos vitais das culturas presentes. A produção de espécies florestais e agrícolas na mesma unidade de terra favorece o produtor e o meio ambiente, harmonizando o agroecossistema com os processos ambientais e podem ser uma grande ferramenta na agricultura familiar e na conservação dos recursos naturais causando uma baixa degradação do meio ambiente.

A atual necessidade de sistemas de produção sustentável coloca em evidência os sistemas agroflorestais, que surgem como alternativa à grande demanda produtiva. A partir da revolução verde na década de 60 a exploração agrícola otimiza as técnicas e formas de manejo na agricultura, e o aumento simultâneo das áreas agricultáveis e da densidade populacional, acaba resultando uma exploração descontrolada de solos e recursos naturais, dessa forma a agricultura convencional apresente-se como um modelo insustentável de produção. A agricultura convencional molda um sistema de produção sustentável, livre de agrotóxicos e de produtos que possam agredir ao meio ambiente, ocasionando um equilíbrio ecológico e conservando características do solo. As técnicas agroecológicas podem servir de auxílio para os agricultores, otimizando as características químicas, biológicas e físicas resultando em uma área com menos processos erosivos, compactação e degradação da matéria orgânica.

\section{Declaração de conflito de interesse}

Nós não temos nenhum conflito de interesse a declarar.

\section{Referências bibliográficas}

Altieri, M. A. (1998). Agroecologia: a dinâmica produtiva da agricultura sustentável (Vol. 1). Porto Alegre, Rio Grande do Sul, Brasil: Editora da Universidade Federal do Rio Grande do Sul. 
Altieri, M. A., Nicholls, C. I., Henao, A. \& Lana, M. A. (2015). Agroecology and the design of climate change-resilient farming systems. Agronomy for Sustainable Development, 35(3), 869-890.

Bandy, D., Garrity, D. P. \& Sánchez, P. (1994). El problema mundial de la agricultura de tala y quema. Agroforestería en las Américas, 1(3), 14-20.

Canuto, J. C. (2017). Sistemas agroflorestais: experiências e reflexões. (8570357095). Brasília, Brasil.

Conway, G. R. (1987). The properties of agroecosystems. Agricultural Systems, 24(2), 95-117.

Daniel, O., Couto, L., Garcia, R. \& Passos, C. A. M. (1999). Proposta para padronização da terminologia empregada em sistemas agroflorestais no Brasil. Revista Árvore, 23(3), 367-370.

EMBRAPA. (2013). Sistema brasileiro de classificação de solos (3a Ed. ed. Vol. 412). Brasíia, DF: Embrapa.

Gliessman, S. R. (2005). Agroecologia - Processos Ecológicos em Agricultura Sustentável. Porto Alegre, Rio Grande do Sul, Brasil: Universdade Federal do Rio Grande do Sul.

King, K. F. \& Chandler, N. T. (1978). The wasted lands: The program of work of the International Council for Research in Agro forestry (ICRAF). Nairobi, Kenya.

Leff, E. (2002). Agroecologia e o saber ambiental. Agroecologia e Desenvolvimento Rural Sustentável, $3,36-51$.

Macedo, R. (2010). Eucalipto em sistemas agroflorestais. Lavras: UFLA.

Mesquita Filho, I. J. D. \& Rocha, E. J. P. L. (2006). Agrofloresta Sucessional: fundamentos, implantação e manejo. Cartilha Ilustrada, 5(2), 1-6.

Oliveira, R. A. \& Oswaldo, T. I. (2012). Agricultura orgânica. Curitiba, Paraná, Brasil: Universidade Federal do Paraná.

Penteado, S. R. (2007). Adubação orgânica: compostos orgânicos e biofertizantes (Vol. 1). Campinas, São Paulo.

Penteado, S. R. (2016). Agricultura alternativa. São Paulo, Brasil: Via Orgânica.

Pereira, R. S. (2004). Poluição hídrica: causas e consequencias. Revista Eletrônica de Recursos Hídricos, 20-36.

Ribaski, J., Montoya, L. J. \& Rodigher, H. R. (2001). Sistemas agroflorestais: aspectos ambientais e socioeconômicos. Informe Agropecuário, 22(212), 61-67.

Rippstein, G., Escobar, G. \& Motta, F. M. (2001). Agroecología y biodiversidad de las sabanas en los Llanos Orientales de Colombia (Vol. 1). Bogotá: CIAT.

Santana, L. B. P. \& Forastieri, V. S. (2003). Sistema agroecológico: Uma via alternativa e Futura.

Stoate, C., Báldi, A., Beja, P., Boatman, N. D., Herzon, I., Van Doorn, A., . . Ramwell, C. (2009). Ecological impacts of early 21st century agricultural change in Europe-a review. Journal of Environmental Management, 91(1), 22-46.

Valeri, S. V., Nóbrega, A. M. F. \& Barreto, V. C. M. (2003). Manejo e florestamento de áreas de preservação permanente e fragmentos florestais. Jaboticabal, São Paulo, Brasil: Funep.

Recebido: 17 dezembro, 2018.

Aprovado: 7 janeiro, 2019.

Publicado: 18 fevereiro, 2019.

Licenciamento: Este artigo é publicado na modalidade Acesso Aberto sob a licença Creative Commons Atribuição 4.0 (CC-BY 4.0), a qual permite uso irrestrito, distribuição, reprodução em qualquer meio, desde que o autor e a fonte sejam devidamente creditados 